\title{
3-Manifold Spines and Bijoins
}

\author{
LUIGI GRASSELEI
}

ABSTRACT. We describe a combinatorial algorithm for constructing all orientable 3-manifolds with a given standard bidimensional spine by making use of the idea of bijoin ([BG]. [Gr]) over a suitable pseudosimplicial triangulation of the spine.

\section{INTRODUCTION}

Throughout this paper, all spaces and maps are piecewise-linear ( $\mathrm{pl}$ ) in the sense of [Gl] or [RS]; all 3-manifolds are supposed to be compact, connected and orientable.

If $M$ is a 3-manifold with non-empty boundary, then a bidimensional polyhedron $K$ such that $M$ collapses to $K$ is said to be a spine of $M$ : if $M$ is closed, a spine of $M$ is a spine of $M-B, B$ being an open 3-ball in $M$.

Given a group presentation $\Phi=\left\{x_{1}, \ldots, x_{g} \mid r_{1}, \ldots, r_{s}\right\}$, denote by $K_{\phi}$ the bidimensional complex constructed as follows:

-- $K_{\Phi}$ has only one O-cell (vertex);

-- the 1-cells (resp. the 2-cells) of $K_{\Phi}$ are in one-to-one correspondence with the generators (resp. the relators) of $\Phi$; denote them by $\alpha_{j}$ (resp. $\left.\beta_{i}\right)$;

each 2-cell $\beta_{i}$ is attached to the 1-skeleton by the formula given by the corresponding relator $r_{j}$.

$K_{\Phi}$ is said to be the standard complex associated to $\Phi$; of course, the factor group of $\Phi$ is $\Pi_{1}\left(\left|K_{\Phi}\right|\right)$. We will not distinguish between a relator $r_{j}$ and any

\footnotetext{
Work performed under the auspices of the G.N.S.A.G.A. of the C.N.R. (National Research Council of Italy) and within the project «Geometria delle Varieta' Differenziabilin of the M.P.I. (Italy).

1980 Mathematies Subject Classification (1985 revision): 57N10, 57M25.

Editorial de la Universidad Complutense. Madrid, 1990.
} 
cyclic conjugate of it or its inverse, since the associated complexes are the same. The above construction may be obviously reversed and each standard complex $K$ induces a group presentation $\Phi_{K}$ of the fundamental group $\Pi_{1}(|K|)$.

It is well known that every 3-manifold $M$ has a standard spine $K_{\Phi}$, for some group presentation $\Phi$, and the factor group of $\Phi$ is clearly $\Pi_{1}(M)$; nevertheless, not every standard complex $K_{\downarrow}$ is a spine of a 3-manifold. Every group presentation $\Phi$ such that $K_{\Phi}$ is a spine of a 3-manifold (resp. of a closed 3-manifold) is said to be geometric (resp. sirongly geometric).

In [N] Neuwirth gives an algorithm for testing if a balanced group presentation (same number of generators and relators) is strongly geometric. The same algorithm is restated by Osborne and Stevens ([OS, ], [S]) by making use of a graph-theoretical tool, the presentation-graph or P-graph $P_{\Phi}$, which can be associated, by a one-to-one correspondence, to the standard complex $K_{\Phi}$ of a group presentation $\Phi$. Namely, $P_{\Phi}$ is essentially the boundary of a regular neighbourhood of the unique vertex of $K_{\Phi}$ and it is easy to prove that $\Phi$ is strongly-geometric if and only if a planar imbedding condition on $P_{\Phi}$ holds. Moreover, as pointed out by Montesinos in [M], a Heegaard diagram of a 3-manifold $M$ gives rise to such a planar imbedding of the $P$ graph $P_{\Phi}$ associated to a suitable group presentation $\Phi$ of $\Pi_{1}(M)$; in fact, this is nothing else than the Whitehead graph of the group presentation $\Phi$ of $\Pi_{1}(M)$ coming from the given Heegaard diagram of $M$. Thus, a group presentation $\Phi$ is geometric (resp. strongly-geometric) if and only if there exists a Heegaard diagram of a 3-manifold (resp. closed 3-manifold) $M$ whose associated presentation for $\Pi_{1}(M)$ is $\Phi$.

In [M] Montesinos describes an algorithm for checking if a given group presentation is geometric; such an algorithm seems to be completely different from Neuwirth's one, since it makes use of branched covering techniques.

In the present paper, we give a combinatorial algorithm for obtaining all 3-manifolds with a given standard spine $K_{\phi}$ by making use of the bijoin construction ([BG], $[\mathrm{Gr}]$ ) applied to a graph-theoretical structure representing a pseudosimplicial triangulation of $K_{\phi}$. This construction allows us to unify, in a common geometric description, both Neuwirth algorithm and Montesinos one; namely, the necessary and sufficient conditions for the geometricity (or the strong-geometricity) of a group presentation obtained in [N], [OS $\mathrm{OS}_{1}$, $\left[\mathrm{OS}_{2}\right],[\mathrm{S}],[\mathrm{M}]$ can be all derived from the bijoin construction.

\section{EDGE-COLOURED GRAPHS AND ASSOCIATED COMPLEXES}

The term pseudograph includes loops and multiple edges, while a multigraph (or simply a graph) allows multiple edges only. 
A (generalized) coloration on a pseudograph $\Gamma=(V(\Gamma), E(\Gamma))$ is a map $\gamma: E(\Gamma) \rightarrow \Delta_{n}=\{0,1, \ldots, n\}$; if $\Gamma$ is a graph, $\gamma$ is said to be proper if $\gamma(e) \neq \gamma(f)$, for each pair $e, f$ of adjacent edges. For each $\gamma \subset \Delta_{n}$, set $\Gamma_{\gamma}=\left(V(\Gamma), \gamma^{-1}(\xi)\right)$; each connected component of $\Gamma$, is often called an

$\%$ residue. For each $i \epsilon \Delta_{n}$, set $i=\Delta_{n}-\{i\}$.

The pair $(\Gamma, \gamma), \Gamma$ being a graph and $\gamma: E(\Gamma) \rightarrow \Delta_{n}$ a (generalized) coloration, is said to be an n-dimensional crystallized structure ([G]) if, for each $i \epsilon \Delta_{n}$, the $\{i\}$-residues are cliques (complete graphs). If all these cliques are of order two, i.e. if $\gamma$ is proper and $\Gamma$ is regular of degree $n+1,(\Gamma, \gamma)$ is simply called an $(n+1)$ - coloured graph $([\mathrm{F}])$.

An $n$-dimensional pseudocomplex $K$ is an $n$-dimensional ball complex in which every $h$-ball, considered with all its faces, is isomorphic with the complex underlying an $h$-simplex; for this reason, each $h$-ball of $K$ is called $h$-simplex. The disjoined star $\operatorname{Std}(s, K)$ of a simplex $s$ in $K$ is defined to be the disjoint union of the $n$-simplexes of $K$ containing $s$, with reidentification of the $(n-1)$-faces containing $s$ and of their faces; the subcomplex Lkd $(s, K)=$ $\{\tau \in \operatorname{Std}(s, K) \mid \tau \cap s=\phi\}$ is called the disjoined link of $s$ in $K$.

As shown in [G] and [F], every $n$-dimensional crystallized structure $(\Gamma, \gamma)$ represents a homogeneous $n$-dimensional pseudocomplex $K(\Gamma)$ constructed by the following rules:

- take an $n$-simplex $\sigma(v)$ for each $v \in V(\Gamma)$ and label its vertices by $\Delta_{n}$;

-- if $v, w \in V(\Gamma)$ are joined by an i-coloured edge, identify the $(n-1)-$ faces of $\sigma(v)$ and $\sigma(w)$ opposite to the vertices labelled by $i$, so that equally labelled vertices are identified together.

Every $h$-simplex $s$ of $K(\Gamma)$, whose vertices are labelled by the distinct colours $c_{1}, \ldots, c_{h} \in \Delta_{n}$, corresponds to a unique $\left(\Delta_{n}-\left\{c_{o}, \ldots, c_{h}\right\}\right)$-residue $\mathfrak{R}$ of $(\Gamma, \gamma)$ and viceversa; its associated pseudocomplex $K(\mathcal{R})$ is Lkd $(s, K(\Gamma))$.

Moreover, $(\Gamma, \gamma)$ is an $(n+1)$ - coloured graph if and only if $|K(\Gamma)|$ is a closed pseudomanifold ([ST]), which is orientable if and only if $\Gamma$ is bipartite ([CGP]).

The construction of $K(\Gamma)$ gives a coloration on the vertex set $S_{o}(K)$ of $K(\Gamma)$ by means of $n+1$ colours (i.e. a map $\xi: S_{v}(K) \rightarrow \Delta_{n}$ which is injective on each simplex of $K(\Gamma)$ ). Given a homogeneous n-dimensional pseudocomplex $K$ with such a coloration on its vertex set $S_{o}(K)$, the construction can be easily reversed yielding an $n$-dimensional crystallized structure, denoted by $\Gamma(K)$.

It is easy to see that $\Gamma(K(\Gamma))=(\Gamma, \gamma)$; moreover (see $[\mathrm{G}]), K(\Gamma(K))=K$ if and only if $K$ satisfies the following property:

$\left(^{*}\right)$ the disjoined star $\operatorname{std}(s, K)$ of every simplex $s$ of $K$ is stronglyconnected. 
A homogeneous $n$-dimensional pseudocomplex satisfying $\left({ }^{*}\right)$ and admitting a coloration on its vertex set by means of $n+\mathrm{l}$ colours is said to be a representable $n$-pseudocomplex, since it is uniquely represented by an $n$ dimensional crystallized structure.

An $n$-dimensional crystallized structure $(\Gamma, \gamma)$ (or its associated pseudocomplex $K(\Gamma)$ ) is said to be contracted if $\Gamma_{i}$ is connected, for each $c \in \Delta_{n}$ (i.e. if $K(\Gamma)$ has exactly $n+1$ vertices). A contracted $(n+1)$-coloured (bipartite) graph $(\Gamma, \gamma)$ is said to be a crystallization of a closed (orientable) $n$-manifold $M$ if $|K(\Gamma)|=M$. Every closed $n$-manifold admits a crystallization ([P]).

For a general survey on manifold representation theory by means of edgecoloured graphs, see [FGG], [BM], [V].

\section{THE BIJOIN CONSTRUCTION}

If $\Gamma$ is an oriented pseudograph and $\gamma: E(\Gamma) \rightarrow \Delta_{n}$ is a (generalized) coloration, the pair $(\vec{\Gamma}, \bar{\gamma})$ is called an $n$-dimensional oriented structure ([BG]) if, for every $i \in \Delta_{n}$, the $\{i\}$-residues are elementary oriented cycles, possibly of length one or two.

By deleting all loops in $E(\vec{\Gamma})$ and by replacing, for every i $\epsilon \Delta_{n}$, each elementary oriented $i$-coloured cycle in $\vec{\Gamma}_{[i\}}$ with a clique on the same vertex set, it is easy to associate an $n$-dimensional crystallized structure $(\Gamma, \gamma)$ to every $n$-dimensional oriented structure $(\bar{\Gamma}, \bar{\gamma})$. Of course, there are, in general, many oriented structures associated to a fixed crystallized structure; they can be easily obtained by reversing the above construction. If $(\vec{\Gamma}, \vec{\gamma})$ is an oriented structure associated to the crystallized structure $(\Gamma, \gamma)$, we set $K(\vec{\Gamma})=K(\Gamma)$.

The following construction, given in [BG], allows to obtain an $(n+1)-$ coloured bipartite graph $(B(\vec{\Gamma}), \beta)$ from an $(n-1)$-dimensional oriented structure $(\vec{\Gamma}, \vec{\gamma})$ :

- $V(B(\vec{\Gamma}))=V(\vec{\Gamma}) \times\{0,1\}$

- for every vertex $v \in V(\vec{\Gamma})$, join $(v, 0)$ with $(v, 1)$ by an edge $e$ of $B(\vec{\Gamma})$ and set $\beta(e)=n$;

- if $\vec{e} \epsilon E(\vec{\Gamma})$ and $\vec{e}(0)=v, \vec{e}(1)=w$, then join $(v, 0)$ with $(w, 1)$ by an edge $e^{\prime}$ of $B\left(\Gamma^{\prime}\right)$ and set $\beta\left(e^{\prime}\right)=\gamma(e)$.

Note that the choice of the opposite oriented structure, obtained by reversing the orientation of each $\{i\}$-residue, for every $i \epsilon \Delta_{n-1}$, gives rise to the same graph. The construction in an adapting to the edge-coloured graphs of a standard method for associating a bipartite graph to an arbitrarily given oriented graph $([\mathrm{BHM}])$. The $(n+1)$-coloured graph $(B(\bar{\Gamma}), \beta)$ (and its 
associated pseudocomplex) is said to be the $h$-bijoin over $(\vec{\Gamma}, \vec{\gamma}), h$ being the number of the $\hat{n}$-residues in $(B(\vec{\Gamma}), \beta)$; if $h=1,(B(\vec{\Gamma}), \beta)$ is simply called bijoin.

Given an $(n+1)$-coloured bipartite graph $(\Gamma, \gamma)$, it is easy to (uniquely) construct, for each $i \in \Delta_{n}$, an $(n-1)$-dimensional oriented structure $(\vec{\Gamma}, i \vec{\gamma})$ such that $(B(\vec{\Gamma}), \beta)=(\Gamma, \gamma)([B G])$; thus, every closed $n$-manifold can be obtained as a bijoin over a suitable $(n-1)$-dimensional oriented structure. A refinement of this result, in dimension three, obtained by making use of "normal crystallizations" ([BDG]), is contained in [Gr].

Extending [M], a closed orientable $n$-dimensional pseudomanifold $N$ (triangulated by a pseudocomplex $K$ ) is said to be a singular n-manifold if the disjoined link of each $k$-simplex, $k>0$, is a sphere and the disjoined link of each vertex is a (closed) connected $(n-1)$-manifold. A vertex of $K$ such that its disjoined link is (resp. is not) an (n-1)-sphere is said to be regular (resp. singular).

Every singular $n$-manifold can be obtained by capping off each boundary component of an $n$-manifold by a cone. In the other sense, if $K$ is a pseudocomplex triangulating a singular $n$-manifold $N$ and $W C S_{o}(K)$, let $M(K, W)$ denote the space obtained by removing from the barycentric subdivision $K^{\prime}$ of $K$ the open stars in $K^{\prime}$ of the vertices belonging to $W$; then $W$ contains all singular vertices of $K$ if and only if $M(K, W)$ is an $n$-manifold whose boundary components are $L k\left(v, K^{\prime \prime}\right)$, with $v \in W$.

Note that, in dimension three, the pseudocomplex $K(\mathrm{~T})$ associated to an arbitrary (bipartite) 4-coloured graph $(\Gamma, \gamma)$ always triangulates a singular 3-manifold.

Proposition 1. Let $(T, \gamma)$ be a 4-coloured bipartite graph such that all $\mathrm{c}-$ labelled vertices of $\mathrm{K}(\mathrm{T})$ are regular, for every $\mathrm{c} \epsilon \Delta_{2}$. If $\mathrm{W}$ denotes the set of all 3-labelled vertices of $\mathrm{K}(\Gamma)$ and $\left({ }^{3} \vec{\Gamma},{ }^{3} \vec{\gamma}\right)$ is the 2-dimensional oriented structure such that $\left(\mathrm{B}\left({ }^{3} \vec{\Gamma}\right), \beta\right)=(\Gamma, \gamma)$, then $\mathrm{K}\left({ }^{3} \vec{\Gamma}\right)$ is a spine of the 3-manifold $\mathrm{M}(\mathrm{K}(\Gamma), \mathrm{W})$.

Proof. It directly follows from the bijoin construction that $K\left({ }^{3} \vec{\Gamma}\right)$ is the subcomplex of $K(\Gamma)$ consisting of all simplexes of $K(\Gamma)$ whose vertices are labelled by colours different from 3 . Thus, for a sufficiently small $\epsilon>0$, the $\epsilon$-neighbourhood $\mathfrak{\lambda}_{\epsilon}$ of $K\left({ }^{3} \bar{\Gamma}\right)$ in $K(\Gamma)$ is an $\epsilon$-neighbourhood of $K\left({ }^{3} \vec{\Gamma}\right)$ in $M(K(\Gamma), W)$ too. For the collapsing criterion for regular neighbourhoods ([RS], corollary 3.30), the polyhedron $\left|\mathfrak{\lambda}_{t}\right|=M(K(\Gamma), W$ ) collapses on $\left|\mathrm{K}\left({ }^{3} \vec{\Gamma}\right)\right|$. 
Corollary 2. If $(\Gamma, \gamma)$ is a 4-coloured bipartite graph representing a (closed, orientable) 3-manifold $M$ such that $K(T)$ has a unique 3-coloured vertex (in particular, if $(T, \gamma)$ is a crystallization of $M$ ), then $|K(\vec{\Gamma})|$ is a spine of $M$.

\section{NEUWIRTH ALGORITHM VIA BIJOINS}

Set $N_{k}=\{1,2, \ldots, k\}$.

If $\Phi=\left\{x_{1}, \ldots, x_{g} \mid r_{1}, \ldots, r_{s}\right\}$ is a group presentation, denote by $\lambda\left(x_{i}\right), i \in N_{g}$, the number of occurrences of the generator $x_{i}$ in the relators of $\Phi$ and by $\lambda\left(r_{j}\right), j \in N_{s}$, the length of each relator $r_{j}$; the length $\lambda$ of $\Phi$ is defined by $\lambda=$ $\sum_{j \in N_{\mathrm{s}}} \lambda\left(r_{j}\right)=\sum_{i \in N_{g}} \lambda\left(x_{i}\right)$. For each relator $r_{j}$, take a 2-cell $\beta_{j}$ and triangulate its boundary by "reading" the relator $r_{j}$. Thus, we obtain a complex $H_{j}$ triangulating $\partial \beta_{j}$ with $\lambda\left(r_{j}\right)$ edges, each of which is labelled by a generator and has a suitable orientation. Label each vertex of $H_{b}$ by the colour 0 , take the barycentric subdivision $H_{j}^{t}$ of $H_{j}$ and label all the barycenters by the colour 1 . Note that each oriented $x_{i}$-labelled edge $\alpha$ of $H_{j}$ splits into an ordered pair $\left(\alpha^{-}, \alpha^{+}\right)$of oriented $x_{i}$-labelled edges in $H_{j}^{\prime}$ : more precisely, if $b_{\alpha}, u_{\alpha}, v_{\alpha}$ respectively denote the barycenter, the first and the second endpoint of $\alpha$, the ordered pair $\left(u_{\alpha}, b_{\alpha}\right)$ (resp. $\left.\left(b_{\alpha}, v_{\alpha}\right)\right)$ represents the endpoints of the oriented edge $\alpha^{-}$(resp. $\alpha^{+}$). By starring $\beta_{j}$ from an inner point $C_{j}$ (labelled by the colour 2) over $H_{j}^{\prime}$, we obtain a pseudocomplex $K_{j}$ triangulating $\beta_{j}$ with a coloration on its vertex set by the colours $0,1,2$ (fig. 1). Now, take the disjoint union $\bigsqcup_{j \in N_{s}} K_{j}$ and identify the oriented edges $\alpha^{-}$(resp. $\alpha^{+}$) of its boundary labelled by the same generator so that identified vertices have the same colour. Let $\widetilde{K}_{\phi}$ be the resulting representable 2-pseudocomplex and let $(\Gamma, \gamma)$ be its associated crystallized structure.

The 0-adjacence (resp. 1-adjacence) in $(\Gamma, \gamma)$ induces a fixed-point-free involution $B$ (resp. $A$ ) on the set $V(\Gamma)$ and the set of the vertices belonging to the same $\{2\}$-residue of $(\Gamma, \gamma)$ can be thought of as an orbit of a suitable permutation $C$ on $V(\Gamma)$. These permutations are the homonimous ones associated to $\Phi$ in [N]. The assignment of such a permutation $C$ gives rise to a particular 2-dimensional oriented structure $\left(\vec{\Gamma}_{C}, \bar{\gamma}_{C}\right)$ associated to the crystallized structure $(\Gamma, \gamma)$; in fact, $C$ induces a cyclic ordering in the vertices of each $\{2\}$-residue of $(\Gamma, \gamma)$ which are the only $\{c\}$-residues of order possibly greater than two. Thus, the geometrical meaning of the choice of a particular $C$ is to give an ordering to the 2 -simplexes of $\widetilde{K}_{\Phi}=K(\Gamma)$ containing the same 1 -simplex.

We always assume this ordering system with the property that the two cyclic orderings on the $\lambda\left(x_{i}\right)$ 2-simplexes containing the two distinct 
$x_{i}$-labelled edges of $\tilde{K}_{\Phi}$ are opposite; this is equivalent to require the property $B C=C^{-1} B$ for the permutation $C$. Let $\Omega(\Phi)$ denote the set of all permutations $C$ on $V(\Gamma)$ whose orbits are the sets of vertices belonging to the same $\{2\}$-residue of $(\Gamma, \gamma)$ and such that $B C=C^{-1} B$.

From now on, the symbol $\left|P_{1}, \ldots, P_{n}\right|$ will denote the orbit number of the group generated by the permutations $P_{h}, h \in N_{n}$, acting on the same set.

Note that, for every $C, C^{\prime} \in \Omega(\Phi),|A, C|=\left|A, C^{\prime}\right|$; in fact, the number $|A, C|$ only depends upon $A$ and the orbits of $C$.

The cellular structure of the pseudocomplex $\tilde{K}_{\Phi}$ immediately shows that $\left|K_{\Phi}\right|$ is the quotient of $\left|\widetilde{K}_{\Phi}\right|$ obtained by identifying the 0 -labelled vertices of $\widetilde{K}_{\Phi}$. Moreover, the number of these vertices, i.e. the number of the $\{1,2\}-$ residues in $(\Gamma, \lambda)$, is $|A, C|$. As a consequence, we have:

Proposition 3. The pseudocomplex $\tilde{K}_{\Phi}$ is a (pseudosimplicial) triangulation of the standard complex $K_{\Phi}$ if and only if $|A, C|=I$.

Remark. Given a group presentation $\Phi$, the number of connected components in the associated $P$-graph $P_{\Phi}$ is $|A, C|([N])$; moreover, it is easy to verify that every 3 -manifold admits a standard spine $K_{\Phi}$ such that the associated $P$-graph $P_{\Phi}$ is connected.

Thus, there is no loss of generality in restricting our study to those group presentations for which $|A, C|=I$ and in supposing that $\widetilde{K}_{\Phi}$ triangulates $K_{\Phi}$.

With the above notations and assumptions, let $C$ be a given permutation in $\Omega(\Phi)$ and let $\left(\vec{\Gamma}_{C}, \vec{\gamma}_{C}\right)$ be the 2-dimensional oriented structure associated to $(\Gamma, \gamma)$ and generated by $C$.

Proposition 4. Let $\left(\Gamma_{C}, \gamma_{C}\right)$ be the h-bijoin over $\left(\vec{\Gamma}_{C}, \vec{\gamma}_{C}\right)$ and let Whe the set of all 3-labelled vertices in $K\left(\Gamma_{C}\right)$.

(a) $h=|A C, B C|$;

(b) the space $M\left(K\left(\Gamma_{C}\right), W\right)$ is a 3-manifold, having $K_{\Phi}$ as a standard spine, if and only if $|A C|=\lambda-2 g+2$;

(c) if (b) holds, the Euler characteristic of $K\left(\Gamma_{C}\right)$ is $g-s+h-1$;

(d) if $g=s$ (resp. $g=s+l$ ) and (b) holds, then $\left|K\left(\Gamma_{C}\right)\right|$ is a closed 3-manifold (resp. $M\left(K\left(\Gamma_{C}\right), W\right)$ is the exterior of a knot), having $K_{\Phi}$ as a standard spine, if and only if $|A C, B C|=h=1$.

Proof. If $\mathscr{F}^{2} \subset \Delta_{2}$ (resp. $\mathscr{F} \subset \Delta_{3}$ ), the symbol $g_{r}$ (resp. $g_{y}^{\prime}$ ) will denote the number of $\bar{Z}$-residues in $(\Gamma, \gamma)$ (resp. in $\left(\Gamma_{C}, \gamma_{C}\right)$ ). 

then

Since the number of 2-simplexes in each $K_{j}$ is $2 \lambda\left(r_{j}\right)$ and $\sum_{j \in N_{s}} \lambda\left(r_{j}\right)=\lambda$,

$$
\operatorname{Card}\left(V\left(\Gamma_{C}\right)\right)=2 \cdot \operatorname{Card}(V(\Gamma))=4 \lambda .
$$

Each $\{c\}$-residue $\left(c \epsilon \Delta_{1}\right)$ in $(\Gamma, \gamma)$ is a complete graph of order two and $g_{\{0\}}=g_{\{1\}}=\lambda$; in fact, in each $K_{j}$ there are exactly $\lambda\left(r_{j}\right)$ edges whose end points are labelled by the colours 2 and $c$ and they are faces of exactly two 2 simplexes.

Hence:

$$
\begin{aligned}
& g_{\{0,3\}}^{\prime}=g_{\{0\}}=|B|=\lambda \\
& g_{\{1,3\}}^{\prime}=g_{\{1\}}=|A|=\lambda .
\end{aligned}
$$

For every $i \in N_{g}$, there are exactly two $\{2\}$-residues in $(\Gamma, \gamma)$ which are complete graphs of order $\lambda\left(x_{i}\right)$. In fact, there are exactly two $x_{i}$-labelled edges in $\widetilde{K}_{\Phi}$ and the number of 2 -simplexes of which each $x_{i}$-labelled edge is a face is the number $\lambda\left(x_{i}\right)$ of occurrences of the generator $x_{i}$ in the relators of $\Phi$. Hence:

$$
g_{\{2,3\}}^{\prime}=g_{\{2\}}=|C|=2 g .
$$

Recall that an alternating path in an oriented graph $\bar{\Gamma}$ is a path whose adjacent edges have opposite orientations. In an oriented structure $(\bar{\Gamma}, \bar{\gamma})$, for every pair $h, k$ of distinct colours, a weak $\{h, k\}$-cycle ([BG]) is an alternating cycle of $(\vec{\Gamma}, \bar{\gamma})$ whose edges are alternatively coloured by $h$ and $k$. If $\vec{g}_{h k}$ denotes the number of weak $\{h, k\}$-cycles of $\left(\bar{\Gamma}_{C}, \vec{\gamma}_{C}\right)$, we have $g_{\{h, k\}}=\vec{g}_{h k}$, for each $h, k \in \Delta_{2}$.

The number of the $\{\hat{3}\}$-residues in $\left(\Gamma_{C}, \gamma_{C}\right)$ is the number of the orbits in the permutation group generated by $A B, B C$ and $A C$. Since $A B=(A C)(B C)^{-1}$, we have:

$$
h=g_{3}^{\prime}=|A B, B C, A C|=|A C, B C|
$$

and this proves (a).

If $P(c), c \in \Delta_{2}$, denotes the permutation on $V\left(\bar{\Gamma}_{C}\right)=V(\Gamma)$ induced by the $c$-adjacence, it is easy to see that $\overleftrightarrow{g}_{h k}=\left|P(h) P(k)^{-1}\right|=\left|P(h)^{-1} P(k)\right|=\vec{g}_{k h t}$, for each pair of distinct colours $h, k \in \Delta_{2}$. Thus, the following equalities hold:

$$
\begin{aligned}
& g_{\{0,1\}}^{\prime}=\vec{g}_{01}=\left|A B^{-1}\right|=|A B|=2 s \\
& g_{\{0,2\}}^{\prime}=\vec{g}_{02}=\left|B^{-1} C\right|=|B C|=\lambda \\
& g_{\{1,2\}}^{\prime}=\overleftrightarrow{g}_{12}=\left|A^{-1} C\right|=|A C| .
\end{aligned}
$$


Note that, $|B C|=\lambda$ since $B C=C^{-1} B$ and hence $(B C)^{2}=1$.

For each $j \in N_{s}$, there is one $\{0,1\}$-residue in $(\Gamma, \gamma)$ which is a bicoloured cycle of length $2 \lambda\left(r_{j}\right)$; in fact, the $\{0,1\}$-residues in $(\Gamma, \gamma)$ are in one-to-one correspondence with the inner vertices $C_{j}$ of $\beta_{j}$. Hence:

$$
g_{\hat{2}}^{\prime}=g_{\hat{2}}=|A, B|=s \text {. }
$$

For each $i \in N_{g}$, there is one $\{0,2\}$-residue in $(\Gamma, \gamma)$ with $2 \lambda\left(x_{i}\right)$ vertices, in fact, the $\{0,2\}$-residues in $(\Gamma, \gamma)$ are in one-to-one correspondence with the barycenters in $\partial K_{i}$. Hence:

$$
g_{i}^{\prime}=g_{i}=|B, C|=g \text {. }
$$

Finally, since, as pointed out for the proof of Proposition 3, the 0-labelled vertices in $\widetilde{K}_{\Phi}$ are in one-to-one correspondence with the $\{1,2\}$-residues in $(\Gamma, \gamma)$, the assumption $|A, C|=1$ gives:

$$
g_{\hat{0}}^{\prime}=g_{\overline{0}}=|A, C|=1 \text {. }
$$

Let us now compute the Euler characteristic $\chi\left(K_{d}\right)$ of the pseudocomplex $K_{\dot{d}}=K\left(\left(\Gamma_{C}\right)_{\bar{d}}\right)$, for each $d \epsilon \Delta_{2}$, by making use of the equalities (1) - (8) and by recalling that the number of 2-simplexes (resp. 1-simplexes) in $K_{\dot{d}}$ is Card $\left(V\left(\Gamma_{C}\right)\right)=4 \lambda\left(\right.$ resp. 3 $\left.\operatorname{Card}\left(V\left(\Gamma_{C}\right)\right) / 2=6 \lambda\right)$.

$$
\begin{aligned}
& \chi\left(K_{0}\right)=4 \lambda-6 \lambda+\left(g_{\{1,2\}}^{\prime}+g_{\{1,3\}}^{\prime}+g_{\{2,3\}}^{\prime}\right)=-2 \lambda+(|A C|+\lambda+2 g)=|A C|+2 g-\lambda ; \\
& \chi\left(K_{i}\right)=4 \lambda-6 \lambda+\left(g_{\{0,2\}}^{\prime}+g_{\{0,3\}}^{\prime}+g_{\{2,3\}}^{\prime}\right)=-2 \lambda+(\lambda+\lambda+2 g)=2 g ; \\
& \chi\left(K_{\hat{2}}\right)=4 \lambda-6 \lambda+\left(g_{\{0,3\}}^{\prime}+g_{\{1,3\}}^{\prime}+g_{\{0,3\}}^{\prime}\right)=-2 \lambda+(\lambda+\lambda+2 s)=2 s .
\end{aligned}
$$

As pointed out in section 2, each $\{\hat{d}\}$-residue in the 4-coloured graph $\left(\Gamma_{C}, \gamma_{C}\right)$ represents the disjoined link of the represented $d$-labelled vertex in $K\left(\Gamma_{C}\right)$. Since the equality [6] (resp. [7] ) ) states that the number of $\{2\}$-residues (resp. $\{\hat{1}\}$-residues) in $\left(\Gamma_{C}, \gamma_{C}\right)$ is s (resp. g), the equality $\chi\left(K_{\hat{z}}\right)=2 s$ (resp. $\left.\chi\left(K_{\mathrm{j}}\right)=2 \mathrm{~g}\right)$ proves that the disjoined link of each 2-labelled (resp. 1-labelled) vertex in $K\left(\Gamma_{C}\right)$ is a 2-sphere. Hence all 1-labelled and 2-labelled vertices of $K\left(\Gamma_{C}\right)$ are regular. Moreover, the disjoined link $K_{0}$ of the unique 0 -labelled vertex of $K\left(\Gamma_{C}\right)$ is a 2-sphere if and only if $\chi\left(K_{0}\right)=|A C|+2 g-\lambda=2$, that is if and only if $|A C|=\lambda-2 g+2$. This result, together with Proposition 1, proves (b).

The Euler characteristic computation of $K\left(\Gamma_{C}\right)$ gives:

$$
\begin{aligned}
& \chi\left(K\left(\Gamma_{C}\right)\right)=\left(g_{0}^{\prime}+g_{1}^{\prime}+g_{\hat{2}}^{\prime}+g_{\hat{3}}^{\prime}\right)-\left(g_{\{0.1\}}^{\prime}+g_{\{0,2\}}^{\prime}+g_{\{0,3\}}^{\prime}+g_{\{1,2\}}^{\prime}+g_{\{1,3\}}^{\prime}+g_{\{2,3\}}^{\prime}\right)+ \\
& +\operatorname{Card}\left(E\left(\Gamma_{C}\right)\right)-\operatorname{Card}\left(V\left(\Gamma_{C}\right)\right)= \\
& =(1+g+s+h)-(2 s+\lambda+\lambda+|\mathrm{AC}|+\lambda+2 g)+8 \lambda-4 \lambda=\lambda+h+1-s-g-|A C| .
\end{aligned}
$$

Thus, if (b) holds, $\chi\left(K\left(\Gamma_{C}\right)\right)=g-s+h-1$. 
Finally, if $g=s$ (resp. $g=s+1)$ and (b) holds, $\chi\left(K\left(\Gamma_{C}\right)\right)=h-1$ (resp. $\chi\left(K\left(\Gamma_{C}\right)=h\right)$ and hence $\left|K\left(\Gamma_{C}\right)\right|$ is a closed 3-manifold (resp. $M\left(K\left(\Gamma_{C}\right), W\right)$ is the exterior of a knot) if and only if $h=1$; proposition 1 , corollary 2 and equality [4] complete the proof of (d).

Proposition 5. Let $M$ be a 3-manifold having $K_{\Phi}$ as a standard spine. There exists a permutation $C \in \Omega(\Phi)$ such that $M=M\left(K\left(\Gamma_{C}\right), W\right)$.

Proof. If $\alpha$ is a 1 -simplex of $\widetilde{K}_{\Phi}$, the imbedding of its star st $\left(\alpha, \widetilde{K}_{\Phi}\right)$ in the (arbitrarily oriented) 3-manifold $M$ induces a cyclic ordering of the 2simplexes of $\widetilde{K}_{\Phi}$ containing $\alpha$. Thus, a permutation $C$ on $V(\Gamma)$ or, equivalently, an oriented structure $\left(\vec{\Gamma}_{C}, \vec{\gamma}_{C}\right)$ can be associated to the crystallized structure $(\Gamma, \gamma)$ representing $\tilde{K}_{\phi}$.

Note that the imbedding of $\bar{K}_{\Phi}$ in $M$ directly gives $B C=C^{-1} B$ and hence $C \in \Omega(\Phi)$. Let $\left(\Gamma_{C}, \gamma_{C}\right)$ be the $h$-bijoin over $\left(\vec{\Gamma}_{C}, \vec{\gamma}_{C}\right)$; note that the choice of the opposite orientation in $M$ gives rise to the opposite oriented structure but to the same graph $\left(\Gamma_{C}, \gamma_{C}\right)$, as pointed out in section 3. If $\hat{M}$ denotes the singular 3-manifold obtained by capping off each boundary component of $M$ by a cone, them $\hat{M}=\left|K\left(\Gamma_{C}\right)\right|$ and hence $M=M\left(K\left(\Gamma_{C}\right), W\right), W$ being the set of all 3-labelled vertices in $K\left(\Gamma_{C}\right)$.

If $\Omega^{\prime}(\Phi)$ denotes the subset of $\Omega(\Phi)$ consisting of all $C \epsilon \Omega(\Phi)$ such that $|A C|=\lambda-2 g+2$, then proposition 4 and proposition 5 lead to the following result:

Corollary 6. The complex $K_{\Phi}$ is a standard spine of a 3-manifold $M$ if and only if there exists a permutation $C \in \Omega^{\prime}(\Phi)$ such that $M=M\left(K\left(\Gamma_{C}, W\right)\right.$.

The above result directly produces an effective algorithm for testing the geometricity of a group presentation, extending Neuwirth's one to nonbalanced presentations.

Example. Let $\Phi=\left\langle x, y \mid x^{3} y^{2}\right\rangle$. In this case, $g=2, s=1$ and, with the notations of fig. 1 , the permutations $A, B$ can be written in the following way:

$$
A=(\overline{1} 2)(\overline{2} 3)(\overline{3} 4)(\overline{4} 5)(\overline{5} 1), B=(1 \overline{1})(2 \overline{2})(3 \overline{3})(4 \overline{4})(5 \overline{5}) .
$$
$\{\overline{4}, \overline{5}\}$.

Moreover, the orbits of the permutation $C$ are $\{1,2,3\},\{\overline{1}, \overline{2}, \overline{3}\},\{4,5\}$, 
The choice of $C=\left(\begin{array}{ll}1 & 23\end{array}\right)(\overline{3} \overline{2} \overline{1})(45)(\overline{5} \overline{4}) \in \Omega^{\prime}(\Phi)$ produces the 4-coloured graph $\left(\Gamma_{C}, \gamma_{C}\right)$ drawn in fig. $l$ and $M\left(K\left(\Gamma_{C}\right), W\right)$ is the exterior of the trefoil knot.

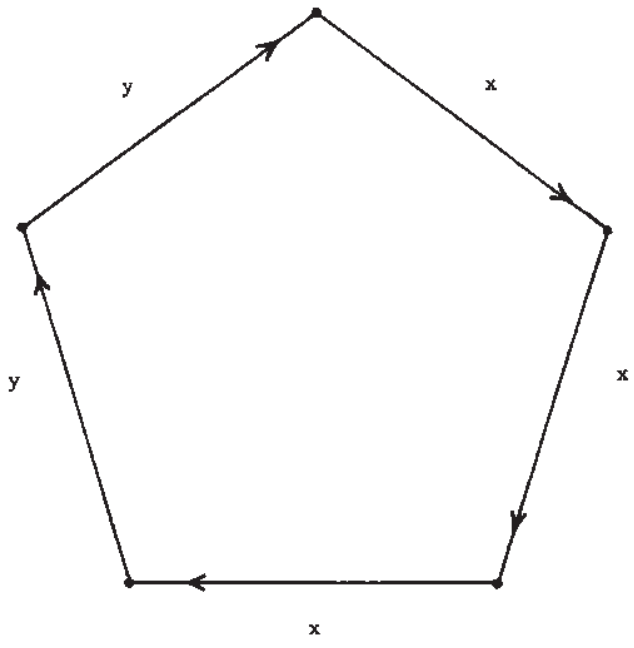

$\mathrm{H}_{1}$

Figure Ia.

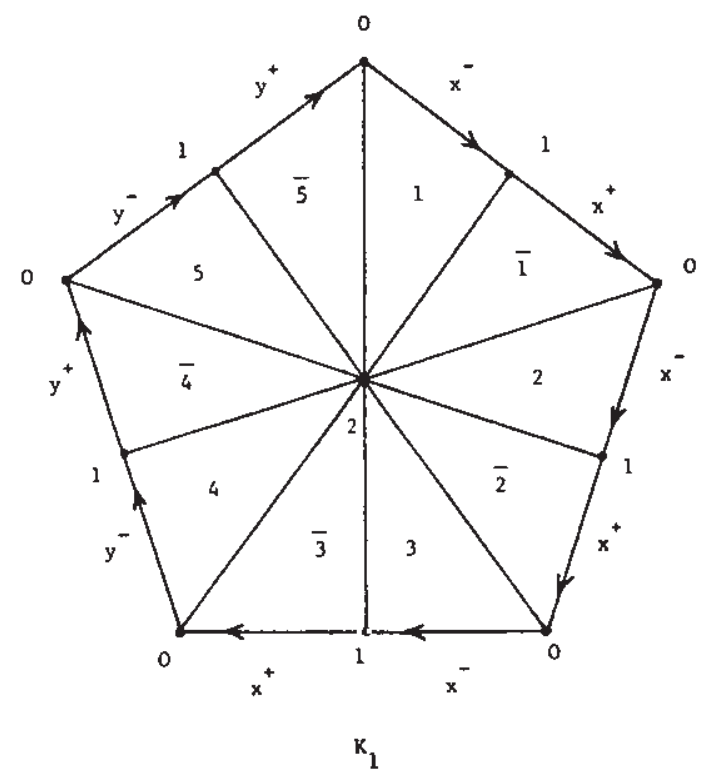

Figure Ib. 


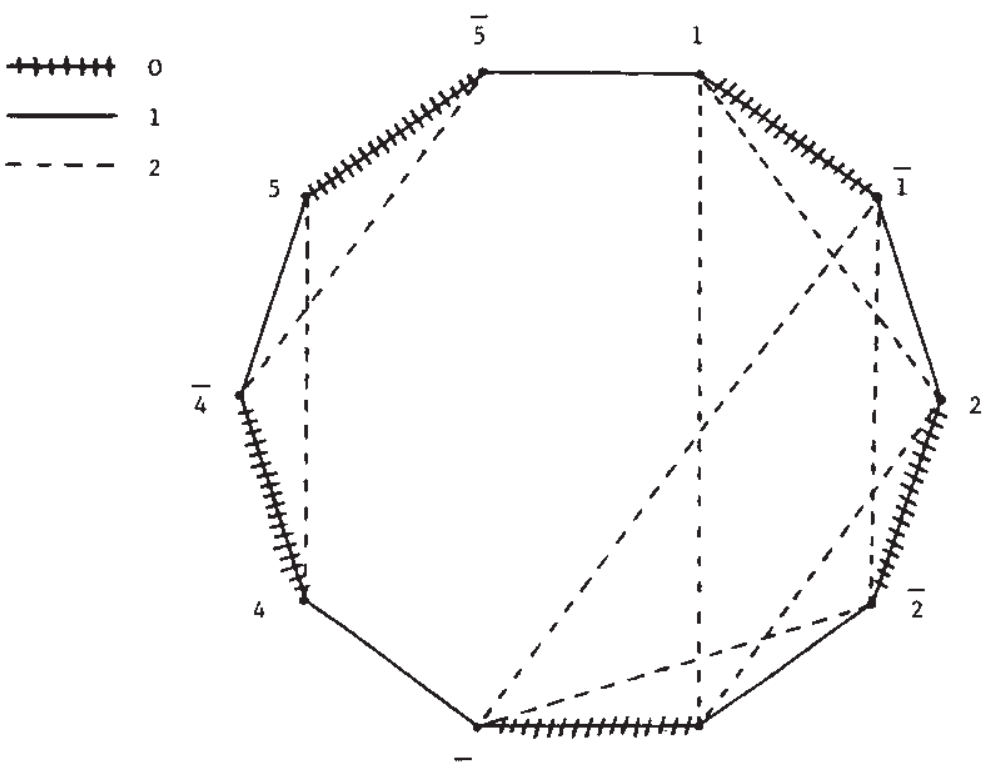

$(\Gamma, \gamma)$

Figure Ic.

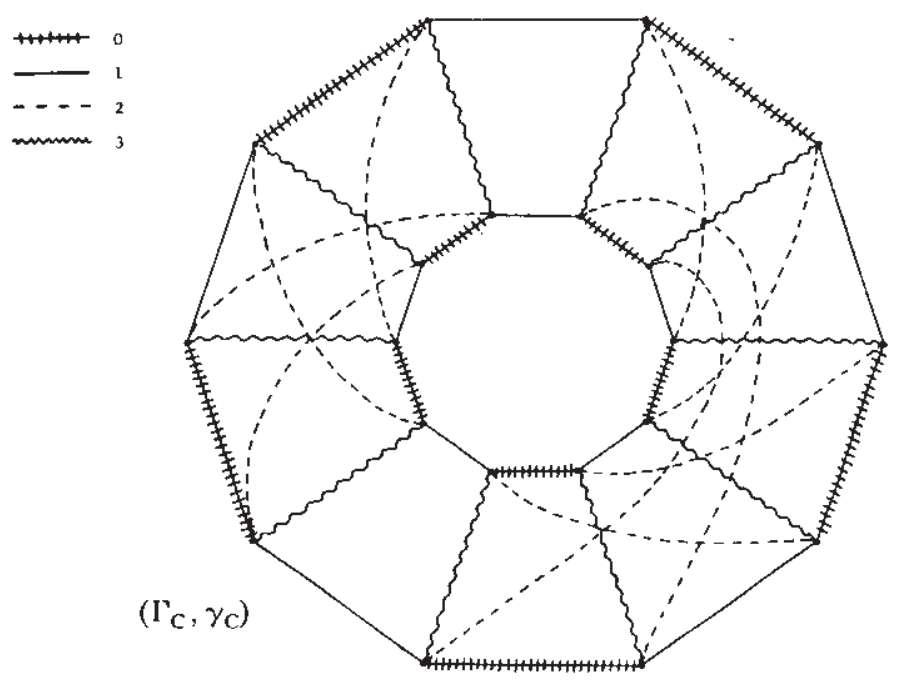

Figure Id. 


\section{MONTESINOS ALGORITHM VIA BIJOINS}

We sketch Montesinos algorithm described in [M].

With the notations of the previous section, let $\Phi$ be a given group presentation whose associated $P$-graph $P_{\Phi}$ is connected; make $\Phi$ positive and call the new presentation $\Phi$ again.

Take the permutation $T=\left(1,2, \ldots, \lambda\left(r_{1}\right)\right) \cdot\left(\lambda\left(r_{\mathrm{I}}\right)+1, \ldots, \lambda\left(r_{1}\right)+\lambda\left(r_{2}\right)\right) . . .5$ $(\ldots, \lambda)$ on $N_{\lambda}$ and the set of all permutations $\sigma$ on $N_{\lambda}$ whose orbits $d_{1}, \ldots, d_{g}$ are defined as follows: the number $j \in N_{\lambda}$ belongs to $d_{i}$ if and only if there is a relator $r_{k}$ whose $\left(j-\lambda\left(r_{k-1}\right)\right)-$ th letter is $x_{i}$. Let $\Sigma(\Phi)$ denote the subset of all such $\sigma$ satisfying $\left|\sigma, \tau \sigma \tau^{-1}\right|=1$ and $|[\sigma, \tau]|=\lambda-2 g+2$.

If $\Sigma(\Phi) \neq \varnothing$, then, for each $\sigma \in \Sigma(\Phi)$, construct the singular 3-manifold $N(\sigma, \tau)$ by taking $\lambda$ copies $\left\{t_{1}, \ldots, t_{\lambda}\right\}$ of the standard tetrahedron $t$ whose bidimensional faces are denoted by $S, \tilde{S}, T, \tilde{T}$. Label the faces $S, \tilde{S}, T, \tilde{T}$ in the copy $t_{i}$ as $S_{i \sigma(i)}, \tilde{S}_{i \sigma-1(i)}, T_{i \tau(i)}, \tilde{T}_{i \tau}{ }^{-1(i)}$ respectively; identify $S_{i j}$ with $\tilde{S}_{j i}$ and $T_{i j}$ with $\tilde{T}_{j s}$ by an orientation-reversing linear homeomorphism respecting the edges $S \cap \tilde{S}$ and $T \cap \tilde{T}$.

If $W$ denotes the set of all singular vertices of $N(\sigma, \tau)$, then $\{M(N(\sigma, \tau), W)$ $\mid \sigma \in \Sigma(\Phi)\}$ is the set of all 3-manifolds $M^{3}$ admitting a Heegaard diagram whose associated presentation for $\Pi_{1}\left(M^{3}\right)$ is $\Phi$.

Since $P_{\Phi}$ is connected, the representable 2-pseudocomplex $\tilde{K}_{\Phi}$ triangulates $K_{\psi}$; now, it is possible to label the vertices of the crystallized structure $(\Gamma, \gamma)$ associated to $\widetilde{K}_{\Phi}$ by the set $\bar{N}_{\lambda}=\{1,2, \ldots, \lambda, \overline{1}, \overline{2}, \ldots, \bar{\lambda}\}$ so that:

$$
\begin{aligned}
& \left.\left.\left.A=(\overline{1} 2) \cdot(\overline{2} 3) \ldots \overline{\left(\lambda\left(r_{1}\right)-1\right.} \lambda\left(r_{1}\right)\right) \cdot \overline{\left(\lambda\left(r_{1}\right)\right.} 1\right) \cdot \overline{\left(\lambda\left(r_{1}\right)+1\right.} \lambda\left(r_{1}\right)+2\right) \ldots \\
& \left.\overline{\left(\lambda\left(r_{1}\right)+\bar{\lambda}\left(r_{2}\right)\right.} \lambda\left(r_{1}\right)+1\right) \text {. } \\
& \therefore\left(\bar{\lambda} \lambda\left(r_{1}\right)+\ldots+\lambda\left(r_{\lambda-1}\right)+1\right) \quad \text { and } \quad B=\mathrm{II}_{h \in N_{\Lambda}}(h \bar{h}) \text {. }
\end{aligned}
$$

Moreover, if $C$ is a permutation on $\bar{N}_{\lambda}$ satisfying the following properties:

- $j$ (resp. $\bar{j}$ ) belongs to the orbit $d_{i}$ (resp. $\bar{d}_{i}$ ) of $C$ if and only if there is a relator $r_{k}$ whose $\left(j-\lambda\left(r_{k-1}\right)\right)$ - th letter is $x_{i}$,

the ordering of the elements $j$ in $d_{i}$ is opposite to the ordering of the elements $\bar{j}$ in $\bar{d}_{i}$,

then $C \in \Omega(\Phi)$.

Thus, the choice of $\sigma$ induces an associated $C_{a}$ (and hence an oriented structure $\left.\left(\vec{\Gamma}_{\sigma}, \vec{\gamma}_{\sigma}\right)=\left(\vec{\Gamma}_{C_{o}}, \vec{\gamma}_{C_{\sigma}}\right)\right)$ in a standard way and viceversa. 
Proposition 7. The singular 3-manifold $N(\sigma, \tau)$ is pl-homeomorphic with $\left|K\left(\Gamma_{\sigma}\right)\right|,\left(\Gamma_{\sigma}, \gamma_{\sigma}\right)$ being the h-bijoin over $\left(\vec{\Gamma}_{\sigma}, \vec{\gamma}_{\sigma}\right)$.

Proof. If $t$ is the standard tetrahedron, subdivide it into four tetrahedra in the following way. If $V_{S}$ (resp. $V_{T}$ ) is the barycenter of $S \cap \tilde{S}$ (resp. $T \cap \tilde{T}$ ), join $V_{S}$ with $V_{T}$ by an edge whose interior is contained in the interior of $t$ and subdivide $S, \tilde{S}$ (resp. $T, \tilde{T}$ ) by joining $V_{S}$ (resp. $V_{T}$ ) with the endpoints of $T \cap \tilde{T}$ (resp. $S \cap \widetilde{S}$ ). Label $V_{S}$ by $1, V_{T}$ by 2 and the endpoints of $S \cap \tilde{S}$ (resp. $T \cap \widetilde{T}$ ) by 0 (resp. 3) (fig. 2).
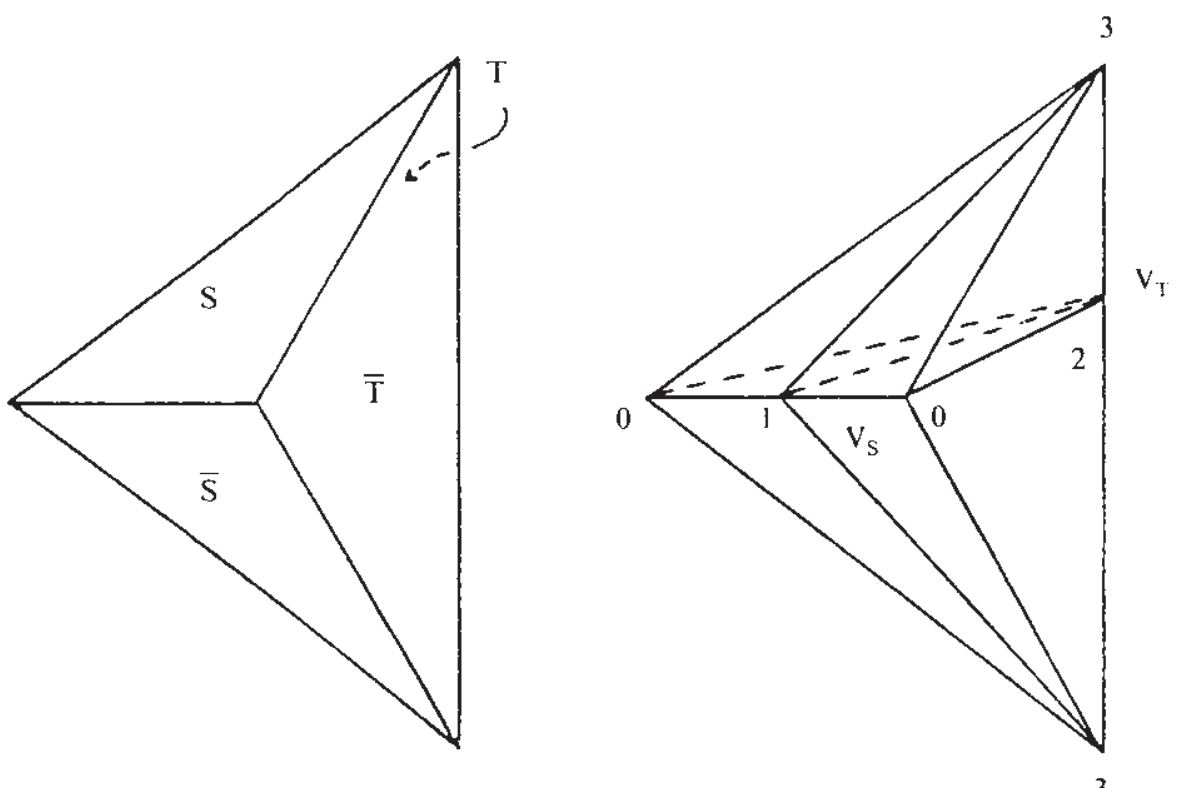

Figure 2

In this way, $N(\sigma, \tau)$ is triangulated by a representable 4-pseudocomplex $K^{\prime \prime}$ in which each $t_{i}$ splits into four tetrahedra.

If $\left(\Gamma^{\prime}, \gamma^{\prime}\right)$ is the 4-coloured graph representing $K^{\prime}$, it is straightforward that the oriented structure $\left({ }^{3} \vec{\Gamma}^{\prime},{ }^{3} \vec{\gamma}^{\prime}\right)$ is isomorphic with $\left(\vec{\Gamma}_{\sigma}, \vec{\gamma}_{\sigma}\right)$ and hence $N(\sigma, \tau)=\left|K\left(\Gamma_{\sigma}\right)\right|$.

Since $\quad[\sigma \sigma, \tau]\left|=g_{\{1,2\}}^{\prime}=\right| A C|, \quad| \sigma|=g, \quad| \tau|=s, \quad| \sigma, \quad \tau \sigma \tau^{-1} \mid=g_{\hat{0}}^{\prime}=$ $|A, C|=1,\left|\tau, \sigma \tau \sigma^{-1}\right|=g^{\prime}=h=|A C, B C|$, all results in [M] can be restated in terms of spines or in terms of bijoins and edge-coloured graphs.

It appears as evident that the graph-theoretical bijoin construction is the idea which unifies both Neuwirth and Montesinos algorithm. 


\section{References}

[BDG] P. BANDiERI-A, DONATI-L. GRASSElli: "Normal» crystallizations of 3-manifolds, Geom. Dedicata 14 (1983), 405-418.

[BG] P. BANDIERI-C. GAGLIARDI: Generating all orientable n-manifolds from (n-1)-complexes, Rend. Circ. Mat. Palermo 31 (1982), 233-246.

[BHM] R. BRUALDi-F. HARARY-Z. MILLER: Bigraphs versus digraphs via matrices, J. Graph Theory 4 (1980), 51-73.

[BM] J. BRACHO-L. MONTEJANO: The combinatorics of colored triangulations of manifolds, Geom. Dedicata 22 (1987), 303-328.

[CGP] A. CavicChioli-L. Grassellu-M. Pezzana: Su una decomposizione normale per le n-varieta' chiuse, Boll. Un. Mat. Ital. 17-B (1980), $1146-1165$.

[F] M. FERRI: Una rappresentazione delle n-varieta' topologiche triangolabili mediante grafi $(n+1)$-colorati, Boll. Un. Mat. Ital. 13-B (1976), 250-260.

[FGG] M. FERRI-C. GaGLIARDI-L. GRASSELl: A graph-theoretical representation of PL-manifolds $=A$ survey on crysiallizations, Aeq. Math. 31 (1986), [2]-141.

[G] C. GAGLIARDI: A combinatorial characterization of 3-manifold crystallizations, Boll. Un. Mat, Ital. 16-A (1979), 441-449.

[GI] L. C. GLASER: Geometrical combinatorial lopology, Van Nostrand Reinhold Math. Studies, New York 1970.

[Gr] L. GRASSELli: A geometric description of "normal" crystallizations, J. Geom. 24 (1985), 36-48.

[M] J. M. MONTESINOS: Representing 3-manifolds by a universal branching set, Math. Proc. Camb. Phil. Soc. 94 (1983), 108-123.

[N] L. P. NEUWIRTH: An algorithm for the construction of 3-manifolds from 2complexes, Proc. Camb. Phil, Soc. 64 (1968), 603-613.

$\left[\mathrm{OS}_{1}\right] \quad$ R. P. OsBorne-R. S. STEVENS: Group presentations corresponding to spines of 3-manifolds I, Amer. J. Math. 96 (1974), 454-471.

[OS $\mathrm{OS}_{2}$ R. P. OSBORNE-R. S. STEVENS: Group presentations corresponding to spines of 3-manifolds II, Trans. Amer. Math. Soc. 234 (1977), 213-243.

[P] M. Pezzana: Sulla struttura topologica delle varieta' compatte, Atti Sem. Mat. Fis. Univ. Modena 23 (1974), 269-277.

[RS] C. P. ROURKE-B. J. SANDERSON: Introduction to piecewise-linear topology, Springer-Verlag, New York-Heidelberg 1972.

[S] R. S. STEVENS: Classification of 3-manifolds with certain spines, Trans. Amer. Math. Soc. 205 (1975), 151-166.

[ST] H. SEIFERT-W. ThRELFALL: Lehrbuch der Topologie, Teubner, Leipzig 1934; English reprint: Academic Press 1980.

[V] A. VincE: $n$-Graphs, Discrete Math. 72 (1988), 367-380.

Dipartimento di Matematica

Piazra Porta San Donato, 5

40127 Bologna Italy 